\title{
Prescribing for transgender patients
}

\section{Louise Tomlins \\ Sexual health physician, Taylor Square Private Clinic, Sydney}

\section{Keywords}

gender dysphoria,

gonadal steroid hormones, transgender persons

Aust Prescr 2019:42:10-3

https://doi.org/10.18873/ austprescr.2019.003

Corrected 4 July 2019

This is the corrected version of the article.

Correction notice available at: https://doi.org/10.18773/ austprescr.2019.053

\section{SUMMARY}

With greater awareness and social acceptance, increasing numbers of transgender individuals are presenting for gender-affirming hormone treatment. There should be a full psychosocial assessment by an experienced clinician before hormone treatment is considered.

People assigned as males at birth who transition to female gender are transgender females. Their management includes an estrogen plus an anti-androgen.

People assigned as females at birth who transition to male gender are transgender males. Their hormone treatment is testosterone.

Treatment is titrated so that hormone concentrations are equivalent to the physiological concentrations of the preferred gender. This aims to minimise adverse effects.

\section{Introduction}

Transgender individuals experience incongruity between their sense of gender and their assigned sex at birth. Psychological distress resulting from this incongruity is known as gender dysphoria.

Increasing numbers of transgender individuals are presenting for medical care, probably because of gradually increasing societal acceptance and awareness. Most individuals will have their care started by specialists in transgender health. General practitioners play a valuable role in providing support and managing comorbidities and may contribute to ongoing treatment and monitoring. Transgender people who are comfortable talking to their GP are likely to have better physical and mental health.

\section{Background}

No formal estimate of the Australian transgender population is available, but a recent study of high school students in New Zealand found they represented $1 \%$ of the population studied.' A typical presentation cannot be described, as the journey taken by each individual is highly variable. No specific age group or cultural or socioeconomic background has been associated with an increased likelihood of presentation.

Some transgender individuals elect not to undergo medical treatment, feeling that a change in gender role or expression is, of itself, sufficient. However, many require masculinising or feminising hormones and in some cases surgery to address the psychological distress of their gender dysphoria.
Many decades of experience with gender-affirming hormone treatment have been accumulated and several guidelines published. ${ }^{2-5}$ Although prescribing remains off label, treatments are relatively straightforward and safe, resembling those used for postmenopausal women or hypogonadal men.

\section{Treatment in adults}

The World Professional Association for Transgender Health advises that medical treatment should only occur after a thorough psychosocial assessment has been undertaken by a clinician experienced in the field. Informed consent must be obtained from the patient. ${ }^{2}$ Standard treatment in adults is based on a gender-affirming hormone. This is testosterone for female to male transition and estrogen, supplemented by an anti-androgen, for male to female transition.

Treatment of transwomen (male to female) produces variable changes including:

- breast growth

- softening of the skin

- reduction and fining of body hair

- change in body fat distribution

- reduced muscle mass and strength in the upper body

- emotional change

- decline in libido

- decreased spontaneous erections

- testicular shrinkage and cessation of spermatogenesis. 
The effects of testosterone on transmen (female to male) are:

- deepening of the voice

- increased muscle mass and strength (particularly upper body)

- change in body fat distribution

- increased hair growth (body and face)

- increase in skin oiliness and body odour

- atrophy of breasts, vulval and vaginal tissues

- clitoral enlargement

- cessation of menstruation.

In both genders, changes begin to appear in the first few months of treatment and usually reach a maximum after three to five years. Starting treatment after puberty will reverse or regress many primary and secondary sexual characteristics, but obviously some will persist to the extent that reassignment surgery might also be sought by some individuals.

\section{Transwomen}

Estrogens in combination with an anti-androgen are the standard first-choice gender-affirming hormone treatments for transwomen.

\section{Estrogen}

Estradiol is preferred, as it most closely resembles the hormone produced by the ovaries. It is prescribed in a similar way to hormone replacement therapy for postmenopausal women, but with slightly higher doses. The dose of estradiol valerate tablets starts at 2-4 mg daily, increasing up to $8 \mathrm{mg}$. Tablets can be given in divided doses if nausea occurs at higher doses.

Patches or implants are preferred for transwomen over 40 years of age (although they can be used in younger people) to minimise the risk of venous thromboembolism. Treatment with patches starts with 100 microgram/24 hours titrated up to 400 micrograms. Implants of 50 and $100 \mathrm{mg}$ are available from compounding pharmacies. Generally, $100 \mathrm{mg}$ is inserted for most transwomen, but a supplementary $50 \mathrm{mg}$ implant can be added for patients with a high body mass index. The duration of drug delivery with implants is on average 6-12 months. Estrogen concentrations should be monitored and a new implant inserted when they fall below physiological levels. Tachyphylaxis can develop with long-term implant use.

Ethinylestradiol and conjugated equine estrogens are generally avoided. They have an increased risk of venous thromboembolism and measurement of their blood concentrations is inaccurate.

\section{Anti-androgens}

Anti-androgens suppress the production and effect of endogenous androgens and so reduce masculine characteristics. In combination with estrogen they reduce the estrogen dose required to achieve feminising effects. The most commonly used antiandrogens are cyproterone and spironolactone.

Cyproterone is a synthetic progestogen with a potent anti-androgenic effect. The usual starting dose is 25-50 mg daily (but it can be started at $12.5 \mathrm{mg}$ ) and can then be increased to $100 \mathrm{mg}$ daily. Rare cases of fulminant hepatotoxicity have been reported with cyproterone use for treatment of metastatic prostate cancer.

Spironolactone is a potassium-sparing diuretic which in higher doses directly inhibits testosterone production and blocks androgen receptors. The usual starting dose is $100 \mathrm{mg}$ daily in one or two doses up to a maximum of $400 \mathrm{mg}$ daily. Blood pressure and potassium concentrations need to be monitored. Possible adverse effects include polyuria, polydipsia and postural hypotension, particularly at higher doses. Hyperkalaemia is also possible, particularly in patients with impaired kidney function or taking potassiumretaining drugs such as ACE inhibitors.

\section{Progesterone}

Progesterone is used by some clinicians in addition to estrogens and anti-androgens. Anecdotal reports suggest that progesterone may improve breast development, but there are no well-designed studies of its use in transwomen. Potential adverse effects include depression, weight gain and an increase in lipids.

\section{Transmen}

Testosterone is used for masculinising effects for transmen. Menstruation typically ceases in the first 3-6 months of treatment. In cases where this is delayed or distress arises from menstruation, progesterone can be used.

\section{Testosterone}

Testosterone is available in a range of formulations, but injected testosterone is the standard first choice. Tablets are unsuitable for gender affirmation as they are unlikely to achieve physiological concentrations or supress menstruation.

\section{Intramuscular injections}

Testosterone enantate was, until recently, commonly used, however it is currently unavailable. It is given by intramuscular injection every 2-3 weeks. Suitable patients can be taught to self-inject. The starting dose is $125 \mathrm{mg}$ titrated up to $250 \mathrm{mg}$ with the aim of reaching male physiological concentrations. There 
can be cyclical effects of aggression or an expansive mood at the start of the cycle and fatigue and irritability at the end.

Injections of testosterone undecanoate $1000 \mathrm{mg}$ every 10-12 weeks produce similar cyclical effects but with less frequency. This formulation carries a risk of pulmonary microembolism, making it unsuitable for self-injection.

\section{Gels and creams}

Testosterone gels and creams are less commonly used, for reasons of practicality. Patients cannot bathe or swim for six hours following application and must avoid contaminating women and children by direct contact. However, for patients who wish to avoid injections or are bothered by their cyclical effects, topical testosterone is a good choice. The standard daily dose is one $50 \mathrm{mg} / 5 \mathrm{~g}$ sachet or 4 actuations of the pump (12.5 $\mathrm{mg}$ per actuation) rubbed into the skin of the upper body. Titrate up to $100 \mathrm{mg}$ (2 sachets or 8 actuations). A testosterone cream is also available, with similar dosing. It comes with an applicator which has $0.5 \mathrm{~mL}$ gradations.

\section{Patches}

Although there is evidence that testosterone patches may eventually achieve similar masculinising effects as injectable formulations, they take a significantly longer time to reach physiological concentrations. For this reason, injectables or topical gels are preferred.

\section{Monitoring}

Hormone concentrations are monitored at threemonthly intervals after treatment begins, to assist with titrating the dose to achieve physiological concentrations for the preferred gender. This is based on a presumption that physiological concentrations will produce maximal effectiveness without undue harm. After the first year, monitoring can be reduced to 6-12-monthly.

In transwomen the target range is equivalent to mid-cycle estradiol concentrations of approximately 400-700 pmol/L. ${ }^{3}$ Testosterone is also monitored in transwomen to ensure suppression to equivalent female concentrations. Some clinicians monitor prolactin concentrations, as a theoretical risk of prolactinoma exists with estrogen treatment.

In transmen, the target range for testosterone is 8-30 mmol/L. Full blood counts should be monitored in case of polycythaemia. The male, rather than female, haemoglobin reference range should be used. ${ }^{6}$ In both genders, blood pressure, weight, renal and liver function are also regularly monitored. Lipids and blood glucose should be monitored in patients with risk factors.
Transmen should continue to undergo cervical and breast screening as long as they still retain these tissues. Prostate cancer screening should also be discussed with transwomen.

\section{Treatment in children}

The treatment of young people is best undertaken by a multidisciplinary team specialised in the area. GPs play an important supporting role for the child and their family. The treatment varies according to the child's stage of puberty. No hormone therapy is required until children reach Tanner Stage 2.

\section{Stage 1 (puberty suppression)}

In stage 1 treatment is reversible. A gonadotrophinreleasing hormone analogue $(\mathrm{GnRH})$ will pause the development of secondary sex characteristics. This allows time for the young person to attain cognitive ability and the maturity required to consider consent for stage 2. Should the child change their mind, stage 1 treatment can be discontinued. The default physiology will then resume the development of the characteristics of the child's sex at birth.

In transfemales the ideal time to commence puberty suppression is at the Tanner Stage 2 of puberty. Anti-androgens can be added to minimise male sexual characteristics if a $\mathrm{GnRH}$ analogue is started at a later stage of puberty.

In transmales the best results are achieved by starting a GnRH analogue at Tanner Stage 2-3. This will suppress menstruation if menarche has already occurred. However, if treatment begins after menarche, norethisterone will achieve the same results and is cheaper and less invasive than injecting a $\mathrm{GnRH}$ analogue.

For both genders, bone health requires attention during stage 1 treatment. Encourage calcium intake and weight-bearing exercise, and monitor bone density and vitamin D concentrations.

\section{Stage 2 (gender-affirming)}

Gender-affirming hormone treatment entails the introduction of either estrogen or testosterone to develop the secondary sex characteristics of the preferred gender and minimise those of the birth gender. The timing depends on the cognitive maturity of the adolescent and their ability to consent to the potentially irreversible effects of treatment. Assessment of this is complex, and requires the full cooperation of the adolescent, their family and treatment team. The potential impacts of treatment delay on the child's health and social functioning must also be considered. 


\section{Conclusion}

Gender-affirming hormone therapy in transgender patients follows similar principles to the treatment of postmenopausal women and hypogonadal men. It is safe if monitored appropriately and delivered with attention to the holistic care of the patient. Specialist care is currently limited and mainly concentrated in metropolitan centres. A supportive GP with knowledge in this area is invaluable to the ongoing care of transgender patients. $<$

\section{Conflict of interest: none declared}

\section{REFERENCES}

1. Clark TC, Lucassen MF, Bullen P, Denny SJ, Fleming TM, Robinson EM, et al. The health and well-being of transgender high school students: results from the New Zealand adolescent health survey (Youth'12). J Adolesc Health 2014;55:93-9. https://doi.org/10.1016/j.jadohealth.2013.11.008

2. Coleman E, Bockting W, Botzer M, Cohen-Kettenis P, DeCuypere G, Feldman J, et al. Standards of care for the health of transsexual, transgender, and gender-nonconforming people, version 7. Int J Transgenderism 2012;13:165-232. https://doi.org/10.1080/15532739.2011.700873

3. Hembree WC, Cohen-Kettenis PT, Gooren L, Hannema SE, Meyer WJ, Murad MH, et al. Endocrine treatment of genderdysphoric/gender-incongruent persons: an Endocrine Society clinical practice guideline. J Clin Endocrinol Metab 2017:102:3869-903. https://doi.org/10.1210/jc.2017-01658

\section{FURTHER READING}

Barrett J. Gender dysphoria in adults. BMJ Best Practice. Last updated Mar 2018. Last reviewed Oct 2018.

https://bestpractice.bmj.com/topics/en-gb/992 [cited 2019 Jan 3]
4. Telfer MM, Tollit MA, Pace CC, Pang KC. Australian standards of care and treatment guidelines for trans and gender diverse children and adolescents. Version 1.1. Melbourne: The Royal Children's Hospital; 2018. www.rch.org.au/adolescentmedicine/gender-service [cited 2019 Jan 3]

5. Deutsch MB, editor. Guidelines for the primary and genderaffiriming care of trangender and gender nonbinary people. 2nd ed. San Francisco: UCSF Center of Excellence for Transgender Health; 2016. http://transhealth.ucsf.edu/ trans?page $=$ guidelines-home [cited 2019 Jan 3]

6. Roberts TK, Kraft CS, French D, Ji W, Wu AH, Tangpricha V, et al. Interpreting laboratory results in transgender patients on hormone therapy. Am J Med 2014:127:159-62. https://doi.org/10.1016/j.amjmed.2013.10.009 